\title{
Analysis of Background Ionospheric Effects on Geosynchronous SAR Imaging
}

\author{
Yifei JI, Qilei ZHANG, Yongsheng ZHANG, Zhen DONG \\ College of Electronic Science and Engineering, National University of Defense Technology, \\ No. 109 De Ya Road, 410073, Changsha, China \\ jyfNUDT@163.com, zhangqilei@nudt.edu.cn \\ Submitted April 28, 2016 / Accepted October 22, 2016
}

\begin{abstract}
Background ionospheric propagation effects are adverse to the performance of Geosynchronous Synthetic Aperture Radar (GEO SAR) system. This paper focuses on the background ionospheric phase advance, which can be modelled as a function of slant total electron content (STEC). The dispersive feature of the phase advance caused by the background ionosphere could be able to distort the GEO SAR range-imaging. Furthermore, for GEO SAR, the integration time is ultra-long and the coverage is ultra-large, thus temporal and spatial distributions of the background ionosphere have to be taken into account. The resultant ionospheric phase variations might decorrelate the azimuth signal and then lead to azimuthimaging deteriorations. In this paper, the theoretical model of the background ionospheric effects on GEO SAR imaging is established and in-depth analyses are presented. Finally, theoretical analyses are validated by the signallevel simulation.
\end{abstract}

\section{Keywords}

Geosynchronous Synthetic Aperture Radar (GEO SAR), imaging, background ionosphere, Total Electron Content (TEC), Slant Total Electron Content (STEC)

\section{Introduction}

Geosynchronous Synthetic Aperture Radar (GEO SAR) runs in an inclined geosynchronous orbit with the altitude of about $36000 \mathrm{~km}$, and thus has advantages of wider swath and short repetition cycle [1-3]. Generally, the operating frequency of GEO SAR is assumed to be within the low-frequency band, e.g., the L-band [2] or S-band [3], which implies that the ionospheric propagation effect can be significant and should be properly studied [4], [5]. The ionosphere is a particular medium with complicated temporal and spatial distributions. A key parameter of the ionosphere is the total electron content (TEC), which is defined as the column density of free electrons. The unit of TEC is $1 \times 10^{16}$ electrons $/ \mathrm{m}^{2}$.
Generally, in terms of spatial distributions, the ionospheric TEC distributions can be categorized into three classes: large-scale, medium-scale and small-scale distributions [6]. Large-scale TEC distributions generally refer to macroscopic scales much larger than the SAR image size. The main error caused by large-scale TEC distributions is a dispersive phase advance. The resulting imaging distortion appears at the range direction, including displacement and defocusing [7]. Medium-scale inhomogeneities refer to the TEC slope, with a spatial scale of several kilometers. The resultant gradient phase error across the azimuth chirp will cause the target displacement. Small-scale disturbances are commonly known as the ionospheric irregularities, which result in scintillation effects. Due to space limitations, this paper refers only to the background ionosphere, which involves the large-scale and medium-scale TEC distributions.

In addition to spatial distributions, the considerable temporal distribution of the background ionosphere exists. In fact, since the integration time of GEO SAR is ultralong, i.e., up to one thousand seconds, the temporal distribution of the background ionosphere has attracted great attentions. Generally speaking, it means that the TEC value varies substantially with different seasons and solar activities. However, for SAR imaging, the concerning issue is the TEC variation during the integration time. It has been indicated that the presence of correlated Gaussian noise was employed to characterize the ionospheric temporaldecorrelation in GEO SAR system [8]. Polynomial approximation was applied to analyze the effect of temporal variations of the background ionosphere [9-12]. Based on this method, an in-depth analysis of the temporal variation of the background ionosphere on GEO SAR imaging was conducted [13].

This paper focuses on the background ionospheric phase advance, which can be modelled as a function of slant total electron content (STEC). STEC is defined as the integral result of the density of free electrons along the signal traversing path. This implies that the variation of the signal traversing path should also be taken into account, besides temporal and spatial distributions of the background ionospheric TEC. Therefore, the analysis should be 
based upon an accurate signal model considering all of these factors.

The aim of this paper is to establish theoretical model and present a comprehensive analysis of the background ionospheric effects on GEO SAR imaging. In Sec. 2, an accurate signal model of GEO SAR imaging influenced by the background ionosphere is developed. Then, the background ionospheric effect on GEO SAR imaging is grouped into two aspects: the effect of the range-imaging and the effect of the azimuth-imaging, which are discussed in Sec. 3 and 4, respectively. Finally, a signal-level simulation is performed to validate the theoretical derivation in Sec. 5.

\section{The Signal Model of GEO SAR Influenced by the Background Ionosphere}

Compared with the Low Earth Orbit SAR (LEO SAR), GEO SAR runs much higher and is relatively slow. Thereby, it is characterized by long aperture time, large coverage and short repeat time. Usually, the nadir point track of GEO SAR is shaped as "8". As listed in Tab. 1, a group of orbital parameters of GEO SAR is applied in the following simulation. The signal model of GEO SAR can be expressed as:

$$
\begin{aligned}
S_{0}\left(f_{\mathrm{r}}, \eta\right)= & W\left(f_{\mathrm{r}}, \eta\right) \cdot \exp \left\{-\mathrm{j} \frac{\pi f_{\mathrm{r}}^{2}}{K_{\mathrm{r}}}\right\} \\
& \cdot \exp \left\{-\mathrm{j} \frac{4 \pi\left(f_{\mathrm{r}}+f_{\mathrm{c}}\right) R(\eta)}{c}\right\}
\end{aligned}
$$

where $\eta$ is the azimuth instant, $f_{\mathrm{r}}$ is the range frequency, $f_{\mathrm{c}}$ is the center frequency, $c$ is the velocity of light, $K_{\mathrm{r}}$ is the range frequency modulation ratio, $W(\cdot)$ signifies the envelope, and $R(\eta)$ is the distance history. Although the same expression can be obtained for GEO SAR, the difference from LEO SAR is mainly embodied in the distance history. In light of the very long integration time and the curved trajectory, the equivalent model of the linear trajectory for traditional LEO SAR cannot satisfy the case of GEO SAR any longer [14]. Thus, the $n$-order Taylor expansion can be applied in the distance history of GEO SAR, which can be written as

$$
R(\eta)=R_{0}+p_{1} \eta+p_{2} \eta^{2}+\ldots+p_{n} \eta^{n}
$$

where $R_{0}$ is the center distance and $p_{1}-p_{n}$ are the first to $n$th order coefficients. Moreover, $n$ is determined according to the precision and efficiency of imaging. A fourth-order model will be applied in the following derivations, which is generally consistent with most conditions [14], [15]. When GEO SAR signals traverse the ionosphere twice, the phase error will be introduced as

$$
\Delta \phi=-\frac{2 \pi \cdot 2 K}{c f} S T E C
$$

\begin{tabular}{|c|c|}
\hline Semi-major Axis [km] & 42164 \\
\hline Eccentricity & 0 \\
\hline Inclination [deg] & 60 \\
\hline Argument of Perigee [deg] & 64.5 \\
\hline Right Ascension of Ascending Node [deg] & 270 \\
\hline The Argument of Latitude [deg] & 17 \\
\hline
\end{tabular}

Tab. 1. Orbital parameters.



Fig. 1. The geometry configuration of GEO SAR with the background ionosphere.

where $f$ is the signal frequency, $K$ is a constant approximately equal to 40.28 and STEC denotes the slant total electron content along the line of sight.

Assuming that the ionosphere is considered to be a thin layer with an altitude of $H_{\text {iono }}$ [6], Figure 1 illustrates the geometric configuration between GEO SAR and the background ionosphere. In Fig. 1, $H_{\text {radar }}$ is the altitude of GEO SAR, and C is the ionospheric penetration point (IPP) at the azimuth instant. $X_{\text {iono }}$ is the length of the system's synthetic aperture at the height of the ionosphere, which denotes the length of the ionospheric penetration trajectory (IPT) generated by the scan of IPP during the integration time. It is generally accepted that the electron density of the ionosphere reaches a maximum at an altitude of approximately $250 \mathrm{~km}-400 \mathrm{~km}$. Above this height, the electron density quickly declines, and the ionospheric effect can be neglected. Therefore, we assume that the mean height of the thin ionosphere layer is $400 \mathrm{~km}$ [6]. Based on (1) and (3), the signal of GEO SAR influenced by the background ionosphere can be written as

$$
\begin{gathered}
S_{\text {iono }}\left(f_{\mathrm{r}}, \eta\right)=S_{0}\left(f_{\mathrm{r}}, \eta\right) \cdot \exp \left\{-\mathrm{j} \frac{2 \pi \cdot 2 K \cdot \operatorname{STEC}(\eta)}{c\left(f_{\mathrm{r}}+f_{\mathrm{c}}\right)}\right\} \\
\operatorname{STEC}(\eta) \triangleq \operatorname{STEC}(x, y ; \eta)=\operatorname{TEC}(x, y ; \eta) \cdot \gamma(\eta)
\end{gathered}
$$

where $\operatorname{TEC}(x, y ; \eta)$ is the vertical TEC, which temporally and spatially varies with the IPP location and the azimuth instant, and $\chi(\eta)$ is the scaling factor, presenting the ratio between the slant and the vertical propagation paths. Moreover, the scaling factor can be expressed as

$$
\gamma(\eta)=\frac{R_{\mathrm{CP}}(\eta)}{H_{\text {iono }}}
$$


where $R_{\mathrm{CP}}(\eta)$ is the distance from the IPP to the target, which varies with the azimuth instant. It can be observed from (5) that the variation of STEC can be attributed to three factors: temporal variations, spatial inhomogeneities of TEC distributions, and varying propagation paths. Without the loss of generalities, these effects on the azimuthimaging along with the range-imaging will be analyzed separately in the following sections.

\section{Background Ionospheric Effects on GEO SAR Range-imaging}

In this section, all variable factors can be assumed to be constant. Generally, $S T E C_{0}$ can be obtained at the central point of the azimuth instant and IPT, which is denoted as large-scale distributions. Then, the dispersion phase error can be written as

$$
\phi_{\text {iono }}=\phi_{0}+\frac{2 \pi \cdot 2 K \cdot S T E C_{0}}{c f_{\mathrm{c}}^{2}} f_{\mathrm{r}}-\frac{2 \pi \cdot 2 K \cdot S T E C_{0}}{c f_{\mathrm{c}}^{3}} f_{\mathrm{r}}^{2}+\delta \phi_{n} .
$$

The constant $\phi_{0}$ has no influence on the image performance, and thus can be neglected. The residual component $\delta \phi_{n}$ causes spurious sidelobes in the range impulse response function. Its value is generally considered to be much smaller than the former three components, and the effect can be safely neglected, as well. Thus, the analysis emphasis should be put on the first-order term and the second-order term.

- The first-order component: The range shift can be introduced as

$$
\Delta L_{\mathrm{r}}=-\frac{K \cdot S T E C_{0}}{f_{\mathrm{c}}^{2}} .
$$

- The second-order component: The maximum values of the range quadratic phase error (QPE) can be expressed as

$$
Q P E_{\mathrm{r} \max }=\frac{\pi K \cdot S T E C_{0}}{c f_{\mathrm{c}}^{3}} B_{\mathrm{r}}^{2}
$$

where $B_{\mathrm{r}}$ is the range bandwidth. It is necessary to consider the range-imaging defocusing when $Q P E_{\mathrm{rmax}}$ is greater than $\pi / 4$.

\section{Background Ionospheric Effects on GEO SAR Azimuth-imaging}

Based on the fourth-order model of the distance history, the azimuth signal model of GEO SAR in time domain can be written as:

$s_{\text {azimuth }}(\eta)=$

$w_{\mathrm{a}}(\eta) \cdot \exp \left\{-\mathrm{j} \frac{4 \pi\left(f_{\mathrm{r}}+f_{\mathrm{c}}\right) \cdot\left(p_{1} \eta+p_{2} \eta^{2}+p_{3} \eta^{3}+p_{4} \eta^{4}\right)}{c}\right\}$ where $w_{\mathrm{a}}(\cdot)$ is the azimuth envelope. Then, the stationary phase solution corresponding to the azimuth frequency $f_{\eta}$ can be given by

$$
\begin{aligned}
\eta= & -\frac{c f_{\eta}}{4\left(f_{\mathrm{c}}+f_{\mathrm{r}}\right) p_{2}}-\frac{3 c^{2} f_{\eta}^{2} p_{3}}{32\left(f_{\mathrm{c}}+f_{\mathrm{r}}\right)^{2} p_{2}^{3}} \\
& +\frac{c^{3} f_{\eta}^{3}\left(-9 p_{3}^{2}+4 p_{2} p_{4}\right)}{128\left(f_{\mathrm{c}}+f_{\mathrm{r}}\right)^{3} p_{2}^{5}}-\frac{5 c^{4} f_{\eta}^{4}\left(27 p_{3}^{2}-24 p_{2} p_{3} p_{4}\right)}{2048\left(f_{\mathrm{c}}+f_{\mathrm{r}}\right)^{4} p_{2}^{7}} .
\end{aligned}
$$

Substituting (11) into (10), the azimuth signal model in the frequency domain can be expressed as

$$
\begin{gathered}
S_{\text {azimuth }}\left(f_{\eta}\right)=W_{\mathrm{a}}\left(f_{\eta}\right) \cdot \exp \left(\mathrm{j} \phi_{\mathrm{a}}\right), \\
\phi_{\mathrm{a}}=\frac{\pi c}{4\left(f_{\mathrm{c}}+f_{\mathrm{r}}\right) p_{2}} \cdot\left[f_{\eta}+\frac{2\left(f_{\mathrm{c}}+f_{\mathrm{r}}\right) p_{1}}{c}\right]^{2} \\
+\frac{\pi c^{2} p_{3}}{16\left(f_{\mathrm{c}}+f_{\mathrm{r}}\right)^{2} p_{2}^{3}} \cdot\left[f_{\eta}+\frac{2\left(f_{\mathrm{c}}+f_{\mathrm{r}}\right) p_{1}}{c}\right]^{3} \\
+\frac{\pi c^{3}\left(9 p_{3}{ }^{2}-4 p_{2} p_{4}\right)}{256\left(f_{\mathrm{c}}+f_{\mathrm{r}}\right)^{3} p_{2}^{5}} \cdot\left[f_{\eta}+\frac{2\left(f_{\mathrm{c}}+f_{\mathrm{r}}\right) p_{1}}{c}\right]^{4} \\
+O\left(f_{\eta}\right)^{5}
\end{gathered}
$$

where $W_{\mathrm{a}}\left(f_{\eta}\right)$ is the spectrum of the azimuth envelope and $\phi_{\mathrm{a}}$ is the azimuth compression phase. It is certain that the coupling between the azimuth and range frequency will introduce the range cell migration term (RCM), and the secondary range compression term (SRC). However, their value variations introduced by the ionosphere are much smaller than the variation of the azimuth compression term introduced by the ionosphere, which can be neglected when analyzing the background ionospheric effects [13]. Additionally, we can reasonably neglect the range frequency [9]. Furthermore, in an attempt to facilitate the analysis of the background ionospheric effect on GEO SAR azimuth-imaging, Taylor expansion versus the azimuth frequency should be performed. Therefore, the azimuth compression phase can be written as

$$
\begin{aligned}
& \phi_{\mathrm{ac}}=\overbrace{\left[\frac{\pi p_{1}}{p_{2}}+\frac{3 \pi p_{1}{ }^{2} p_{3}}{4 p_{2}{ }^{3}}+\frac{\pi p_{1}{ }^{3}\left(9 p_{3}{ }^{2}-4 p_{2} p_{4}\right)}{8 p_{2}{ }^{5}}\right]}^{P_{1}} f_{\eta} \\
& +\overbrace{\left[\frac{\pi c}{4 f_{\mathrm{c}} p_{2}}+\frac{3 \pi c p_{1} p_{3}}{8 f_{\mathrm{c}} p_{2}{ }^{3}}+\frac{\pi c p_{1}{ }^{2}\left(27 p_{3}{ }^{2}-12 p_{2} p_{4}\right)}{32 f_{\mathrm{c}} p_{2}{ }^{5}}\right]}^{P_{2}} f_{\eta}^{2} \\
& P_{3} \\
& +\overbrace{\left[\frac{\pi c^{2} p_{3}}{16 f_{\mathrm{c}}{ }^{2} p_{2}{ }^{3}}+\frac{\pi c^{2} p_{1}\left(9 p_{3}{ }^{2}-4 p_{2} p_{4}\right)}{32 f_{\mathrm{c}}{ }^{2} p_{2}{ }^{5}}\right]}^{p_{\eta}} f_{\eta}^{3} \\
& +\frac{\overbrace{c^{3}\left(9 p_{3}^{2}-4 p_{2} p_{4}\right) \pi}^{P_{4}}}{256 f_{\mathrm{c}}^{3} p_{2}{ }^{5}} f_{\eta}^{4}
\end{aligned}
$$


where $P_{n}, n=1,2,3,4$ are the coefficients of the azimuth phase versus the azimuth frequency. On account of (4), (12) and (14), the azimuth signal model in the frequency domain influenced by the background ionosphere can be presented as

$$
S_{\text {a_iono }}\left(f_{\eta}\right)=F\left\{F^{-1}\left[S_{\text {azimuth }}\left(f_{\eta}\right)\right] \cdot \exp \left[-\mathrm{j} \frac{2 \pi \cdot 2 K \cdot \operatorname{STEC}(\eta)}{c f_{\mathrm{c}}}\right]\right\}
$$

In (15), $F$ denotes the Fourier transform, which can be efficiently evaluated via the fast Fourier transform (FFT). As mentioned in Sec. 2, the background ionospheric effects on the GEO SAR azimuth-imaging attribute vary with the STEC, which can be divided into three categories: temporal variations, spatial variations of the background ionosphere, and varying traversing-paths. In the subsequent sections, three dedicated analyses are presented.

\subsection{The Effect of Temporal Variations of the Background Ionosphere}

In this section, the background ionosphere is assumed to be spatially homogeneous and the scaling factor is assumed to be constant. We only discuss the effect of temporal variations of the background ionosphere. In general, the temporally varying STEC can be expanded with respect to the azimuth time as

$$
\operatorname{STEC}(\eta)=\operatorname{STEC}_{0}+k_{1} \eta+k_{2} \eta^{2}+k_{3} \eta^{3}+\delta \operatorname{STEC}(\eta)
$$

where $S T E C_{0}$ is the constant component, which has no effect on azimuth-imaging and can be ignored. $\delta \operatorname{STEC}(\eta)$ is the residual component, and can also be neglected due to its much smaller value. Moreover, $k_{1}-k_{3}$ are the first-order, second-order and third-order coefficients with units of TECU/s, TECU $/ \mathrm{s}^{2}$ and TECU $/ \mathrm{s}^{3}$, respectively. Compared with LEO SAR, the integration time of GEO SAR is quite long, reaching $10^{2}-10^{3}$ seconds. Therefore, STEC cannot be constant within the integration time, and the azimuthimaging degradation resulting from the temporally varying STEC must be taken into account. Based on (14), (15) and (16), the azimuth compression phase influenced by temporal variations of the background ionosphere can be presented as

$$
\begin{aligned}
& \phi_{\mathrm{ac}}=\overbrace{\left[\frac{\pi p_{1}^{\prime}}{p_{2}^{\prime}}+\frac{3 \pi p_{1}^{\prime 2} p_{3}^{\prime}}{4 p_{2}^{\prime 3}}+\frac{\pi p_{1}^{\prime 3}\left(9 p_{3}^{\prime 2}-4 p_{2}^{\prime} p_{4}\right)}{8 p_{2}^{\prime 5}}\right]}^{P_{1}^{\prime}} f_{\eta} \\
& +\overbrace{\left[\frac{\pi c}{4 f_{\mathrm{c}} p_{2}^{\prime}}+\frac{3 \pi c p_{1}^{\prime} p_{3}^{\prime}}{8 f_{\mathrm{c}} p_{2}^{\prime 3}}+\frac{\pi c p_{1}^{\prime 2}\left(27 p_{3}^{\prime 2}-12 p_{2}^{\prime} p_{4}\right)}{32 f_{\mathrm{c}} p_{2}^{\prime 5}}\right]}^{P_{2}^{\prime}} f_{\eta}^{2} \\
& +\overbrace{\left[\frac{\pi c^{2} p_{3}^{\prime}}{16 f_{\mathrm{c}}^{2} p_{2}^{\prime 3}}+\frac{\pi c^{2} p_{1}^{\prime}\left(9 p_{3}^{\prime 2}-4 p_{2}^{\prime} p_{4}\right)}{32 f_{\mathrm{c}}^{2} p_{2}^{\prime 5}}\right]}^{P_{2}^{\prime}} f_{\eta}^{3}+\overbrace{\frac{\pi c^{3}\left(9 p_{3}^{\prime 2}-4 p_{2}^{\prime} p_{4}\right)}{256 f_{\mathrm{c}}^{3} p_{2}^{\prime 5}}}^{P_{2}^{\prime}} f_{\eta}^{4}
\end{aligned}
$$

where

$$
p_{n}^{\prime}=p_{n}+\frac{K}{f_{\mathrm{c}}^{2}} \cdot k_{n}, \quad n=1,2,3
$$

It can be observed that $k_{1}, k_{2}, k_{3}$ will introduce linear phase error (LPE), quadratic phase error (QPE), cubic phase error (CPE) and fourth phase error (FPE). Here, the effect of FPE can be reasonably neglected due to its much smaller value. For azimuth-imaging, it is generally accepted that LPE could be able to result in imaging shift.

$$
\Delta L_{\mathrm{a}}=\frac{\left(P_{1}^{\prime}-P_{1}\right)}{2 \pi} v_{\eta}
$$

where $v_{\eta}$ is the ground velocity of the beam footprint. While, QPE might be able to broaden the main lobe and raise sidelobes, and CPE might be able to induce asymmetric sidelobes. Based on (17) and (18), the maximum value of QPE and CPE can be expressed as

$$
\left\{\begin{array}{l}
Q P E_{\eta \max }=\left(P_{2}^{\prime}-P_{2}\right) \cdot B_{\eta}{ }^{2} / 4 \\
C P E_{\eta \max }=\left(P_{3}^{\prime}-P_{3}\right) \cdot B_{\eta}{ }^{3} / 8
\end{array}\right.
$$

where $B_{\eta}$ is the azimuth bandwidth. This means that the size of QPE and CPE depends on both the azimuth bandwidth and the change-rate of the temporally-varying background ionosphere. The azimuth-imaging deterioration is necessary to be considered when QPE exceeds $\pi / 4$ or CPE exceeds $\pi / 8$.

In the following, a group of experimentally measured data of the vertical TEC, provided by the China Research Institute of Radio Wave Propagation, is applied to analyze the effect of temporal variations of the background ionosphere on the azimuth-imaging of the L-band GEO SAR. As shown in Fig. 2, the TEC data, lasting for 24 hours, were measured at $20.0^{\circ} \mathrm{N}, 110.0^{\circ} \mathrm{E}$, and measured on December 15th, 2001, June 15th, 2007, and December 15th, 2007, respectively. It is obvious from Fig. 2 that the vertical TEC varies with measuring time.

It can be found from Fig. 3 that QPE and CPE exceed the threshold from time to time, which implies that the effect of temporal variation of the background ionosphere has to be considered in the GEO SAR azimuth-imaging. Moreover, based on the calculated result in Fig. 3, we may find that:

- QPE and CPE peak at dawn and dusk. This means that the ionospheric turbulence may be severe at these time periods. The reason for this is that the measured TEC consists of both the background ionosphere and the ionospheric irregularities.

- Broadly speaking, QPE and CPE in 2001 are higher than that in 2007 . This may be caused by more active solar-activities in 2001. It was reported that a large number of massive solar flares occurred and the 10.7-cm solar-flux index was much higher in 2001 [6]. 

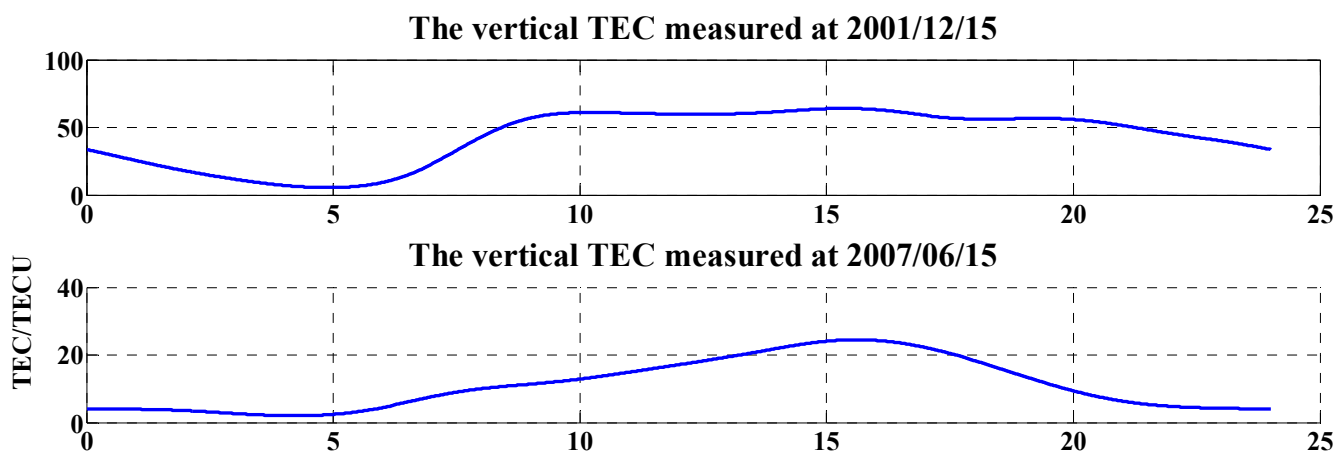

The vertical TEC measured at 2007/12/15

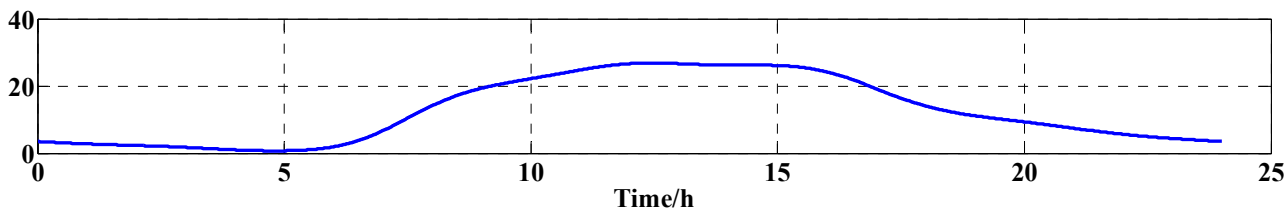

Fig. 2. The experimental measured data of the vertical TEC.


Fig. 3. The azimuth QPE and CPE with the integration time set as ten minutes.

In conclusion, the azimuth-imaging is susceptible to the temporally-varying background ionosphere and its distortion must be taken into account when the azimuth QPE and CPE exceed the reference value.

\subsection{The Effect of Spatial Variations of the Background Ionosphere}

Here, the background ionosphere is assumed to be temporally invariable and the scaling factor is assumed to be constant. We only consider the effect of spatial distributions of the background ionosphere. Generally, the spatially varying STEC can be expressed as

$$
\operatorname{STEC}^{\prime}(x, y, \eta)=\operatorname{TEC}\left(x, y ; \eta_{0}\right) \cdot \gamma\left(\eta_{0}\right) .
$$

As mentioned above, the spatial variation of the background ionosphere mainly refers to the TEC gradient, which can be denoted as $\triangle T E C$ with the unit of TECU/km. Considering the result of the azimuth-imaging shift, another first-order coefficient of STEC is introduced into (16) as

$$
k_{1}^{\prime}=\Delta T E C \cdot \gamma\left(\eta_{0}\right) \cdot V_{\text {iono }}
$$

where $V_{\text {iono }}$ is the velocity of the IPP and can also be calculated by

$$
V_{\text {iono }}=X_{\text {iono }} / T_{\eta} \text {. }
$$

We can use the International Reference Ionosphere (IRI) model to obtain spatially varying TEC data by setting the altitude, the geographic location and the time [16]. The measured area is defined by the longitude ranging from $105.0^{\circ} \mathrm{E}$ to $114.0^{\circ} \mathrm{E}$ and by the latitude ranging from $14.0^{\circ} \mathrm{N}$ to $22.0^{\circ} \mathrm{N}$. The measured time is set to be $17: 00$ UTC on December 15th, 2001. The altitude is chosen to be 


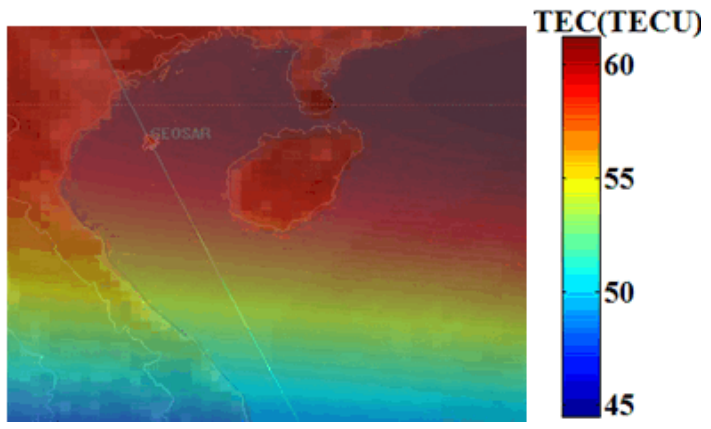

Fig. 4. Spatial distributions of the vertical TEC.

$400 \mathrm{~km}$. Figure 4 shows the geometry characteristic between the nadir point trajectory of the satellite and spatial distributions of the vertical TEC. It is reasonably assumed that the STEC gradient is constant within a synthetic aperture because the IPT length is generally tens of kilometers. Considering that the IPT length is $18.7 \mathrm{~km}$ and the integration time is $600 \mathrm{~s}$, we can obtain the azimuth shift along the nadir point track by using (19). It is shown in Fig. 5 that the spatial-variance of the TEC gradient results in the spatially varying azimuth shift with the movement of the platform, albeit constant within a synthetic aperture.

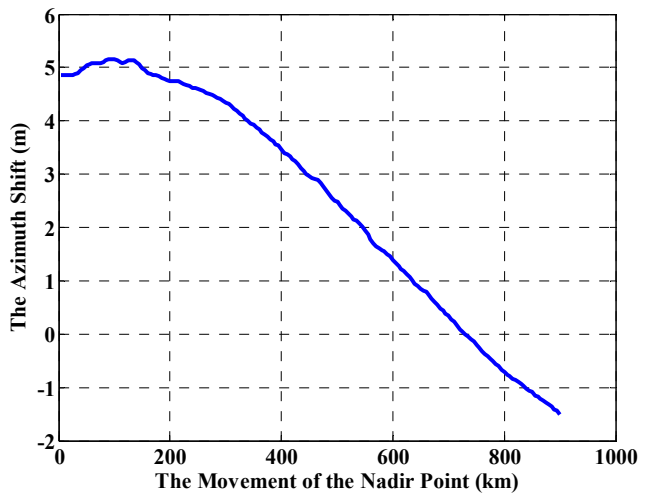

Fig. 5. Spatially varying azimuth shift.
To summarize this section, the spatially varying background ionosphere can introduce the TEC gradient, which causes the considerable azimuth-shift. Furthermore, the TEC gradient is considered to be constant within a synthetic aperture but spatially varying along the satellite track.

\subsection{The Effect of Varying Propagation Paths}

Because the altitude of the GEO SAR is much higher than the background ionosphere, $R_{\mathrm{CP}}(\eta)$, part of the slant range, rather than the entire length, leads to STEC varying with the azimuth time (see Fig. 1). In this section, the background ionosphere is assumed to be spatially and temporally homogeneous, and only the effect of varying propagation paths will be considered. Therefore, equation (5) can be simplified as

$$
\operatorname{STEC}(\eta)=T E C \frac{R_{\mathrm{CP}}(\eta)}{H_{\text {iono }}} .
$$

Considering the coordinate of the satellite and the target in each moment can be accurately obtained, the intersection coordinate between the straight line and the ionospheric layer can be calculated by solving equations; thus, we can obtain the distance of $R_{\mathrm{CP}}(\eta)$ and the varying STEC. The STEC curves are presented in Fig. 6 with different incident angles and vertical TEC values, where the constant part of STEC is removed. We can also use the polynomial approximation method to obtain the additional $k^{\prime \prime}{ }_{1}-k^{\prime \prime}{ }_{3}$ from Fig. 6 , which are summarized in Tab. 2 . The magnitudes of $k^{\prime \prime}{ }_{1}$ and $k^{\prime \prime}{ }_{3}$ are so small that the azimuthshift caused by $k_{1}^{\prime \prime}$ and CPE introduced by $k_{3}{ }_{3}$ can be neglected.

Substituting $k^{\prime \prime}$ for $k_{2}$ in (18), we can obtain the azimuth QPE by using (20). The azimuth QPEs, varying with

\begin{tabular}{|c|c|c|c|c|}
\hline The vertical TEC [TECU] & The incident angle [degrees] & $k^{\prime \prime}{ }_{1}[\mathrm{TECU} / \mathrm{s}]$ & $k^{\prime \prime}{ }_{2}\left[\mathrm{TECU} / \mathrm{s}^{2}\right]$ & $k^{\prime \prime}{ }_{3}\left[\mathrm{TECU} / \mathrm{s}^{3}\right]$ \\
\hline 30 & 15 & $-7.75 \times 10^{-11}$ & $1.05 \times 10^{-7}$ & $-7.24 \times 10^{-13}$ \\
\hline 30 & 30 & $-8.66 \times 10^{-11}$ & $1.15 \times 10^{-7}$ & $-1.74 \times 10^{-12}$ \\
\hline 50 & 30 & $-1.44 \times 10^{-10}$ & $1.91 \times 10^{-7}$ & $-2.90 \times 10^{-12}$ \\
\hline
\end{tabular}

Tab. 2. Coefficients of the temporally varying STEC influenced by varying propagation paths

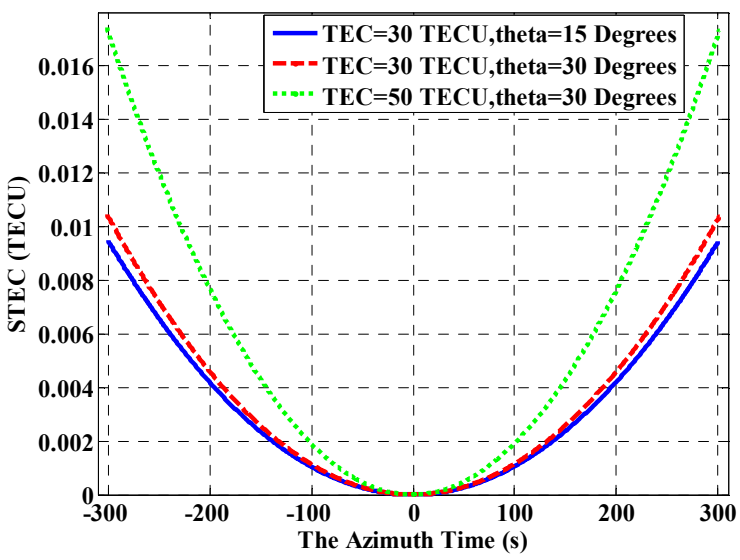

Fig. 6. The STEC curves influenced by varying propagation paths.

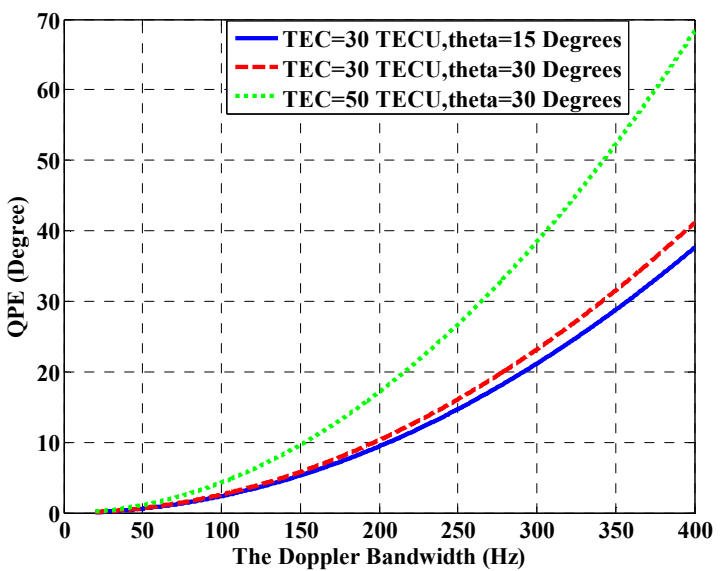

Fig. 7. QPE introduced by varying propagation paths. 
the different Doppler bandwidths, are provided in Fig. 7, which shows that the azimuth-imaging will seriously worsen due to QPE as the vertical TEC, the incident angle and the Doppler bandwidth increase. We can only account for the effect of the second-order coefficient introduced by varying propagation paths.

We can only account for the effect of the secondorder coefficient introduced by varying propagation paths. In addition, the azimuth QPE is a composite result of the vertical TEC, the incident angle and the Doppler bandwidth.

\section{Simulation Results}

In this section, simulations will be conducted to verify the theoretical analysis, including both the effect on the range-imaging and azimuth-imaging. The simulation parameters are listed in Tab. 3. For temporal variations of the background ionosphere, the temporal-varying TEC, measured at $20^{\circ} \mathrm{N}, 110^{\circ} \mathrm{E}$, on December 15,2001 , are applied. In the simulation, the TEC values last for $600 \mathrm{~s}$, i.e., from 17:00 to 17:10 UTC. Meanwhile, spatial variations of the background ionosphere are obtained from the above-mentioned IRI data. It should be noted that only the first-order coefficient of STEC should be considered when simulating the effect of the spatially varying background ionosphere. As discussed in Sec. 4.3, the effect of varying propagation paths has a concrete contribution to the second-order coefficient of STEC. Furthermore, STEC coefficients attributed to each factor are listed in Tab. 4. Based on these radar parameters and the background ionospheric data, simulation of the point target is conducted. In this simulation, the first-order coefficient of STEC is the addition of $k_{1}$ and $k_{1}^{\prime}$, the second-order coefficient of STEC is the addition of $k_{2}$ and $k^{\prime \prime}{ }_{2}$, and the third-order coefficient of STEC is $k_{3}$.

Simulation results of the point target are provided in Fig. 8. It can be observed that the image shift in both the range and azimuth direction arises from the background ionosphere. In addition, the imaging is seriously deteriorated, exhibiting signs of defocusing and the loss of peak power. Compared with ideal imaging, Fig. 9 illustrates both the range and azimuth profiles. In an attempt to intuitively describe the performance of the image, some evaluation indexes of the range-imaging and azimuth-imaging influenced by the background ionosphere are summarized in Tab. 5 .

\begin{tabular}{|c|c|}
\hline Center frequency [GHz] & 1.25 \\
\hline Range bandwidth [MHz] & 100 \\
\hline Doppler bandwidth [Hz] & 180 \\
\hline Integration time [s] & 600 \\
\hline Incident angle [degrees] & 30 \\
\hline Ground velocity [m/s] & 454.1 \\
\hline Length of IPT [km] & 18.7 \\
\hline Range resolution [m] & 1.33 \\
\hline Azimuth resolution [m] & 2.28 \\
\hline
\end{tabular}

Tab. 3. Operating parameters.

\begin{tabular}{|c|c|c|}
\hline (a) Constant component & $\mathrm{STEC}_{0}[\mathrm{TECU}]$ & 66.04 \\
\hline \multirow{2}{*}{$\begin{array}{c}\text { (b) Temporal-varying } \\
\text { background ionosphere }\end{array}$} & $k_{1}[\mathrm{TECU} / \mathrm{s}]$ & $2.02 \times 10^{-3}$ \\
\cline { 2 - 3 } & $k_{2}\left[\mathrm{TECU} / \mathrm{s}^{2}\right]$ & $1.63 \times 10^{-6}$ \\
\cline { 2 - 3 } & $k_{3}\left[\mathrm{TECU} / \mathrm{s}^{3}\right]$ & $1.67 \times 10^{-9}$ \\
\hline $\begin{array}{c}\text { (c) Spatial-varying } \\
\text { background ionosphere }\end{array}$ & $k_{1}^{\prime}[\mathrm{TECU} / \mathrm{s}]$ & $9.45 \times 10^{-4}$ \\
\hline (d) Varying propagation paths & $k_{2}^{\prime \prime}\left[\mathrm{TECU} / \mathrm{s}^{2}\right]$ & $2.52 \times 10^{-7}$ \\
\hline
\end{tabular}

Tab. 4. STEC coefficients attributed to each factor.

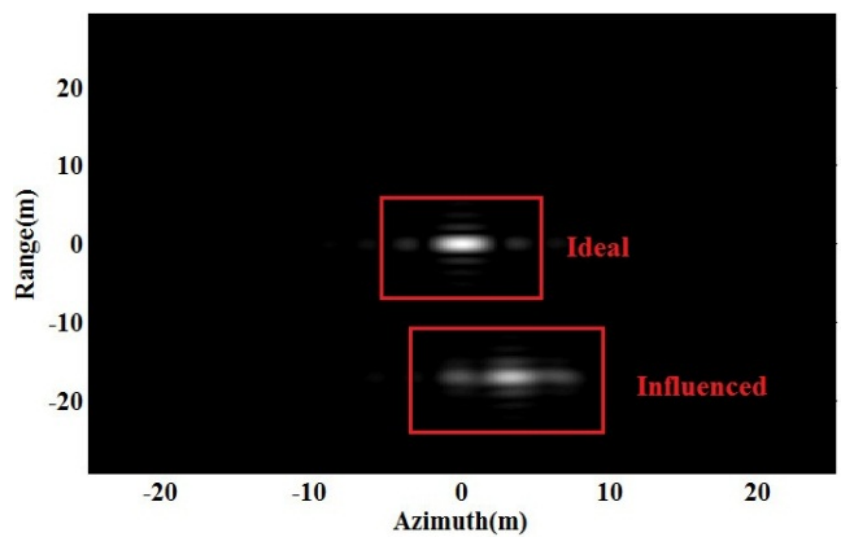

Fig. 8. Simulation results of a point target.

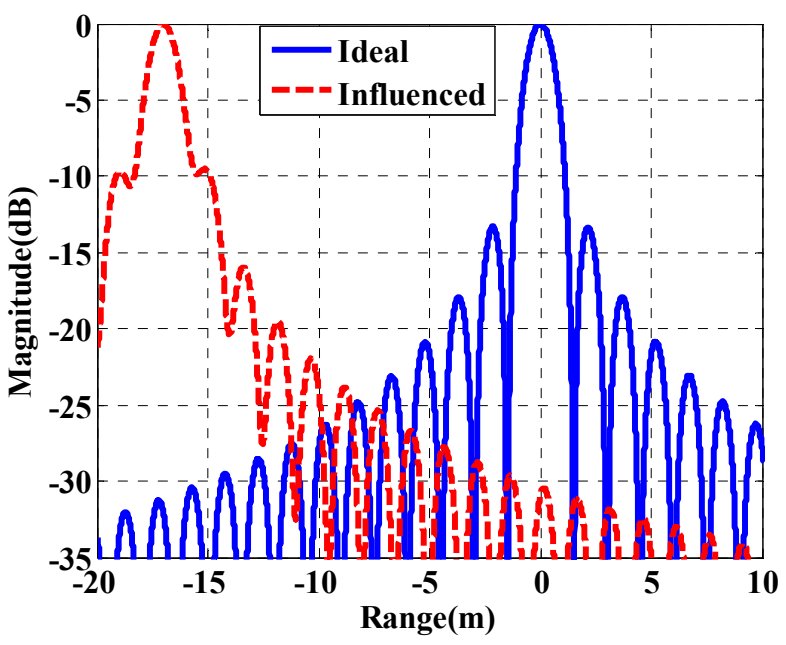

(a)

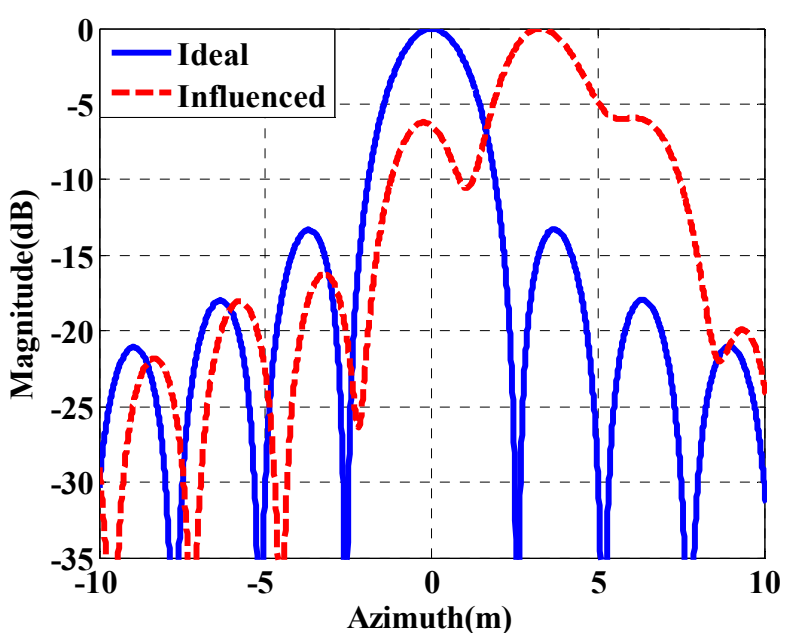

(b)

Fig. 9. Profiles. (a) Range. (b) Azimuth. 


\begin{tabular}{|c|c|c|}
\hline Evaluation Indexes & Range & Azimuth \\
\hline Shift [m] & -17.01 & 3.29 \\
\hline QPE [Degree] & 81.72 & 97.69 \\
\hline CPE [Degree] & - & 33.47 \\
\hline PSLR [dB] & -9.56 & -4.85 \\
\hline ISLR [dB] & -6.81 & -5.67 \\
\hline The Broaden Coefficient & 1.05 & 1.07 \\
\hline
\end{tabular}

Tab. 5. Evaluation indexes.

The deterioration of the range-imaging is serious, for the range QPE is more than 45 degrees; the deterioration mainly includes the broadening of the impulse response width, the increase of the peak side lobes ratio (PSLR) and the integrated side lobes ratio (ISLR). For the azimuthimaging, both QPE and CPE are above the reference values. This indicates that the azimuth-imaging worsens along with the asymmetric side lobes. In addition to the deterioration of the focusing performance, image shifts also arise both in the range and azimuth directions. It is worth mentioning that simulated shifts are consistent with theoretical values computed by (8) and (19).

\section{Conclusions}

The presence of the background ionosphere has an adverse impact on GEO SAR imaging. Considering the curved trajectory of GEO SAR, an accurate signal model influenced by the background ionosphere has been derived. The effects of STEC are divided into the constant and varying components, which distort the range and azimuth image, respectively. For GEO SAR range-imaging, the range shift can be significant, and the deterioration cannot be neglected when the background ionospheric range QPE is more than $\pi / 4$. For azimuth-imaging, varying STEC results in varying phase errors and, consequently, leads to considerable deteriorations. It is shown that the variation of STEC is contributed by temporal-spatial variation of the background ionosphere and varying propagation paths. The temporal-varying background ionosphere plays a leading role in azimuth-imaging deteriorations. An additional azimuth shift can be caused by medium-scale TEC distributions, which are denoted as the TEC gradient variation. Furthermore, the effect of varying propagation paths could be able to result in defocusing. In the near future, the associated compensation method of background ionosphere effects in GEO SAR system should be studied.

\section{Acknowledgment}

The authors sincerely thank the editors and reviewers for their constructive comments. Yifei Ji is also grateful to the China Research Institute of Radio Wave Propagation for their support. This work was supported by the National Nature Science Foundation of China under Grant No. 41271459 .

\section{References}

[1] TOMIYASU, K. Synthetic aperture radar in geosynchronous orbit. In Symposium Digest of the Antennas and Propagation Society International Symposium. Washington (USA), 1978, vol. 16, p. 42-45. DOI: 10.1109/APS.1978.1147948

[2] NASA, JPL. 2003, Global Earthquake Satellite System: a 20-year plan to enable earthquake prediction [EB/OL]. Available: http:// solidearth.jpl.nasa.gov/GESS/3123_GESS_Rep_2003.pdf.

[3] HU, C., LIU, Z. P., LONG, T. An improved CS algorithm based on the curved trajectory in geosynchronous SAR. IEEE Journal of Selected Topics in Applied Earth Observations and Remote Sensing, 2012, vol. 5, no. 3, p. 795-808. DOI: 10.1109/JSTARS.2012.2188096

[4] ISHIMARU, A., KUGA, Y., LIU, J., KIM, Y., FREEMAN, T. Ionospheric effects on synthetic aperture radar at $100 \mathrm{MHz}$ to 2 GHz. Radio Science, 1999, vol. 34, no. 1, p. 257-268. DOI: 10.1029/1998RS900021

[5] XU, Z. W., WU, J., WU, Z. S. A survey of ionosphere effects on space-based radar. Waves in Random Media, 2004, vol. 14, no. 2 , p. 189-273. DOI: 10.1088/0959-7174/14/2/008

[6] MEYER, F., BAMLER, R., JAKOWSKI, N., FRITZ, T. The potential of low-frequency SAR systems for mapping ionospheric TEC distributions. IEEE Geoscience and Remote Sensing Letters, 2006, vol. 3, no. 4, p. 560-564. DOI: 10.1109/LGRS.2006.882148

[7] BELCHER, D. P. Theoretical limits on SAR imposed by the ionosphere. IET Radar, Sonar and Navigation, 2008, vol. 2, no. 6, p. 435-448. DOI: 10.1049/IET-RSN:20070188

[8] BRUNO, D., HOBBS, S. E. Radar imaging from geosynchronous orbit: Temporal decorrelation aspects. IEEE Transactions on Geoscience and Remote Sensing, 2010, vol. 48, no. 7, p. 2924 to 2929. DOI: $10.1109 /$ TGRS.2010.2042062

[9] TIAN, Y., HU, C., DONG, X. C., et al. Theoretical analysis and verification of time variation of background ionosphere on geosynchronous SAR imaging. IEEE Geoscience and Remote Sensing Letters, 2015, vol. 12, no. 4, p. 721-725. DOI: 10.1109/LGRS.2014.2360235

[10] DONG, X. C., HU, C., TIAN, W. M., et al. Design of validation experiment for analysing impacts of background ionosphere on geosynchronous SAR using GPS signals. Electronics Letters, 2015, vol. 51, no. 20, p. 1604-1606. DOI: 10.1049/EL.2015.1545

[11] LI, L., HONG, J., MING, F. Study about ionospheric effects on medium-Earth-orbit SAR imaging. In Proceedings of the IEEE Radar Conference. Cincinnati (OH, USA), 2014, p. 27-31. DOI: 10.1109/RADAR.2014.6875549

[12] JI, Y. F., ZHANG, Q. L., ZHANG, Y. S., YU, A.X., DONG, Z. Analysis of background ionospheric effects on geosynchronous SAR azimuth imaging. In Proceedings of the 11th European Conference on Synthetic Aperture Radar (EUSAR). Hamburg (Germany), 2016, p. 1207-1210. ISBN: 978-3-8007-4228-8 /ISSN: 2197-4403

[13] HU, C., TIAN, Y., YANG, X. P., et al. Background ionosphere effects on geosynchronous SAR focusing: Theoretical analysis and verification based on the BeiDou Navigation Satellite System (BDS). IEEE Journal of Selected Topics in Applied Earth Observations and Remote Sensing, 2016, vol. 9, no. 3, p. 1143-1162. DOI: $10.1109 /$ JSTARS.2015.2475283

[14] LI, D. X., SUN, Z. Y, HE, F., et al. Phase error analysis in GEO SAR imaging based on MSR. In Proceedings of the IET International Radar Conference. Xian (China), 2013, p. 291-295. DOI: $10.1049 / \mathrm{cp} .2013 .0171$ 
[15] LI, D. X., WU, M. Q., SUN, Z. Y, et al. Modeling and processing of two-dimensional spatial-variant geosynchronous SAR data. IEEE Journal of Selected Topics in Applied Earth Observations and Remote Sensing, 2015, vol. 8, no. 8, p. 3999-4009. DOI: 10.1109/JSTARS.2015.2418814

[16] BILITZA, D. The international reference ionosphere Climatological standard for the ionosphere. In Characterising the Ionosphere, Meeting Proceedings RTO-MP-IST-056. Neuilly-surSeine (France), 2006, p. 32-1-32-12. Available from: http://www.rto.nato/abstracts.asp

\section{About the Authors ...}

Yifei JI was born in 1992 and received the M.S. degree in Information and Communication Engineering from the
National University of Defense Technology (NUDT), Changsha, China, in December 2016. Now, he is a doctor's candidate of NUDT. His research interests include synthetic aperture radar and ionosphere.

Qilei ZHANG was born in 1984 and received the B.S. degree in Communication Engineering, the M.S. degree and Ph.D. degree in Information and Communication Engineering from the National University of Defense Technology, Changsha, China, in 2007, 2009 and 2014, respectively. From January 2012 to July 2013, he was a visiting Ph.D. student with the University of Birmingham, Birmingham, UK. He is currently a lecturer with the National University of Defense Technology. His fields of interest include synthetic aperture radar, bistatic synthetic aperture radar and ionosphere. 